\title{
EVM-based Performance Evaluation of Co-channel Interference Mitigation using Spatial Filtering for Digital MIMO Receivers
}

\author{
Sajad Golabighezelahmad ${ }^{1}$, Eric Klumperink ${ }^{2}$, Bram Nauta $^{3}$ \\ IC Design Group, University of Twente, Enschede, The Netherlands \\ ${ }^{1}$ s.golabighezelahmad@utwente.nl, 2e.a.m.klumperink@utwente.nl, ${ }^{3}$ b.nauta@utwente.nl
}

\begin{abstract}
Digital Multiple-Input Multiple-Output (MIMO) receivers, exposed to strong co-channel interference, require high dynamic range Analog-to-Digital Converters (ADCs) and a highly linear RF front-end. Hybrid analog-digital beamforming can mitigate this co-channel interference by spatial filtering before the ADC and improve interference robustness. This paper demonstrates and characterizes this mitigation effect in detail utilizing Error Vector Magnitude (EVM) as a criterion. A 4-element $22 \mathrm{~nm}$ FD-SOI CMOS prototype MIMO Receiver chip is characterized with a linear 4-element dipole antenna array with half-wavelength spacing in the $2.4 \mathrm{GHz}$ ISM-band. EVM measurements demonstrate the effectiveness of spatial filtering for co-channel interference mitigation and dynamic range extension. The receiver is exposed to interference while using different QAM formats (16-QAM to 256-QAM). It is demonstrated how interference reduces the acceptable-EVM input power range, while hybrid beamforming extends this range. This is done by extensive conductive measurements for reliable results, while also demonstrating an Over-the-Air (OTA) result.

Keywords - Spatial filtering, Digital demodulation, Multiple-Input Multiple-Output (MIMO), Error Vector Magnitude (EVM), Constellation diagram, Quadrature Amplitude Modulation (QAM), Interference rejection, Over-the-Air (OTA) Measurement.
\end{abstract}

\section{INTRODUCTION}

Digital MIMO systems have recently found broad application, as they allow for spatial multiplexing and diversity gains. Hence, digital MIMO is favored for efficient use of scarce radio spectrum in sub-GHz bands by improving link throughputs as well as link reliability. However, there are challenges to overcome as the spectrum becomes heavily utilized, so that strong mutual interference occurs. Where a change of WiFi channel in the $2.4 \mathrm{GHz}$ band used to be effective in the past, it is now rarely possible to find an unused channel in urban areas. Hence, co-channel interference needs to be addressed. Typical $2.4 \mathrm{GHz}$ MIMO receivers have e.g. 4 antennas allowing for digital spatial filtering. However, the RF front-ends and ADCs are susceptible to strong in-band interferes which can easily degrade performance (see Fig. 1a).

To alleviate high dynamic range requirements, hybrid beamforming MIMO receiver architectures have been proposed [1], [2], [3]. RF/analog beamforming before the ADCs can reduce interference spatially as shown in Fig. 1b, while identical receive chains without analog beamforming get all exposed to strong interference as in Fig. 1a. Previous work typically demonstrates interference robustness improvement by conductive measurements for one rather arbitrarily chosen scenario. This paper addresses the question how the interference robustness benefit can be characterized for different practical use scenarios, targeting to define and characterize a "good user experience" criterion. We propose to do that in this paper for QAM formats using EVM as a criterion. We will show how EVM varies depending on interference and spatial filtering defining an acceptable-EVM input power range. The paper is structured as follows. In section II we summarize the properties of our hybrid beamforming chip design [3], briefly. In section III we introduce EVM as a performance metric, and model the EVM of our receiver chip using a MATLAB model. Then we verify this model using conductive measurement in section IV and expose the receiver to interference to demonstrate how EVM degrades. We will demonstrate that this reduces the acceptable-EVM input power range and quantify the degradation. Moreover, we will demonstrate how hybrid beamforming increases this power range and validate this by an OTA experiment. Finally, we draw conclusions in section V.

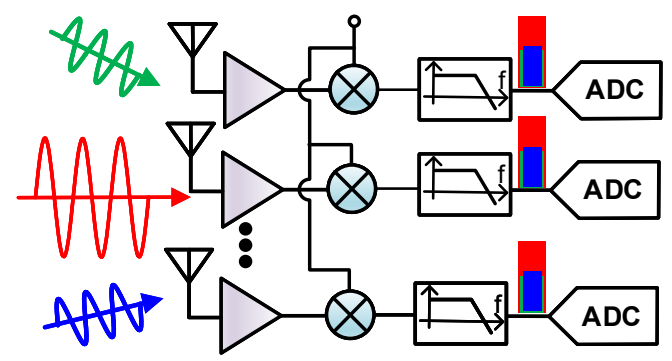

(a)

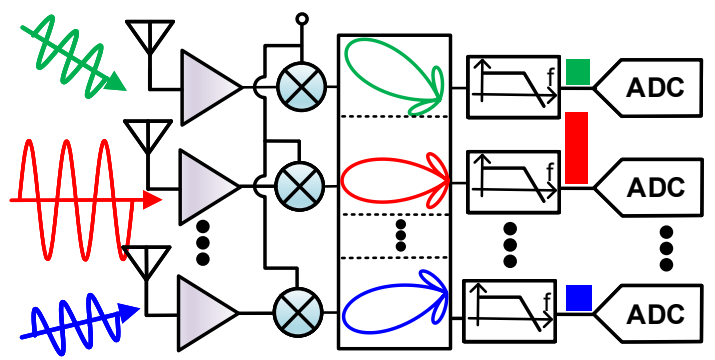

(b)

Fig. 1. (a) Digital MIMO receivers with separate receive chains are susceptible to a strong co-channel interferer; (b) Analog multi-beamforming can reduce this interference while still receiving signals from all directions. 


\section{SPATIAL FILTERING By HYBRID BEAMFORMING RECEIVER}

Our receiver design described in [3] targets hybrid beamforming to improve the dynamic range of the receiver and ADCs by spatial filtering. Unlike traditional Multiple-Input-Single-Output (MISO) systems that only produce a single output beam in a particular direction, we produce as many beams as the number of antennas. The idea is to enable reception from signals from all directions, but manipulate their strength so that strong interferers are suppressed in most of the outputs. As a simple intuitive example, consider a set of beamforming weights that produces a set of orthogonal beams, as shown in bottom part of Fig. 2 , such that one of the beams points in the direction of the strong blocker (red beam). As the beams are orthogonal, the other 3 output beams ideally do not receive the interferer and only one ADC has to handle the strong signal. If we simply throw away this heavily distorted output in the digital domain, a spatial notch filter is realized (see notch in Fig. 2). Of course more smart adaptive approaches are possible ${ }^{1}$, but this example serves to show that 3 of the $N=4$ outputs would now still produce clean output signals in almost all directions (bottom right plot in Fig. 2). In our receiver chip [3], analog weights are realized using a "constant-gm vector modulator" [3], [4]. This architecture also allows for direct current domain beamforming, resulting in a highly linear RF receiver front-end with wideband interference rejection, as detailed in [3].

\section{EVM AS PERFORMANCE EVALUATION METRIC FOR SPATIAL FILTERING}

EVM is defined as root-mean-square (RMS) error of vector differences between measured constellation point and its ideal location, referenced relative to RMS value of constellation vectors, i.e., the EVM is expressed as [5]:

$$
E V M_{r m s}=\sqrt{\frac{\frac{1}{N} \sum_{i=1}^{N}\left(\left|I_{i}-\hat{I}_{i}\right|^{2}+\left|Q_{i}-\hat{Q}_{i}\right|^{2}\right)}{\frac{1}{N} \sum_{i=1}^{N}\left(I_{i}^{2}+Q_{i}^{2}\right)}}
$$

where $N$ is number of symbols received, $I$ and $Q$ are the ideal and in-phase quadrature levels, and $\hat{I}$ and $\hat{Q}$ are the received in-phase and quadrature values. It is typically expressed in \%. EVM is a favorable system-level specification because it can provide useful insights for quality evaluation of digitally demodulated signals, allowing for identification of circuit impairments, e.g., noise and distortion in transceivers. Moreover, EVM provides a bridge between circuit level specifications such as NF, IIP3, etc., and system-level signal quality measurements such as BER, channel capacity or data rate, etc. Reference [6] uses EVM plots to demonstrate performance of a single-chain, reconfigurable dual-band

\footnotetext{
${ }^{1}$ We could also add Automatic Gain Control (AGC) for each ADC and still receive the signal.
}

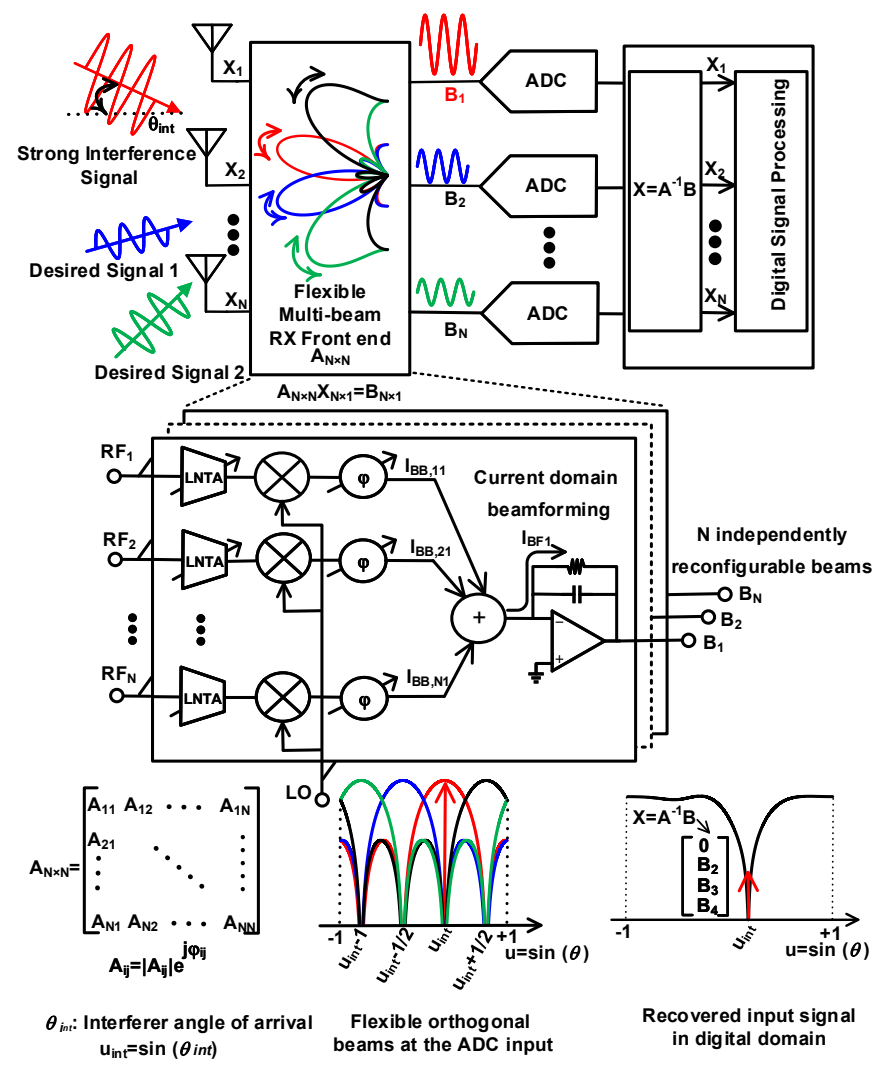

Fig. 2. Proposed hybrid analog-digital MIMO receiver using multiple RF phased arrays. Flexible orthogonal beams for $N=4$ are created to form a notch at interferer's angle-of-arrival [3].

receiver for carrier aggregation in TV bands below 1 GHz. In [7], EVM has been related to coverage range for a 5G millimeter-wave link. In this paper, we use EVM to quantify the adverse effect of in-band interferers in demodulation of small signal-of-interest (SoI) for various demodulation formats, e.g. 256-QAM, 64-QAM, etc., as well as demonstrating benefits of spatial filtering for MIMO receivers. In order to verify SNR improvement of the phased array receiver, the EVM in the noise-limited region can be used to estimate the effective array-receiver noise figure. To do so, the EVM can be related to the SNR in the noise-limited region as [5]:

$$
S N R=-20 \log (\operatorname{EVM}(\%) / 100)
$$

and SNR is simply the difference between signal and noise powers in decibels:

$$
S N R=P_{\text {signal }}-P_{\text {noise }}
$$

where the noise power in $\mathrm{dBm}$ is given by:

$$
P_{\text {noise }}=-174+10 \log (B W)+N F
$$

Therefore, the measured EVM values for a few input power levels at the noise-limited region can be used to estimate the noise figure using curve fitting with varying noise figure values as in [8] or even with a single-point EVM measurement but with less accuracy. It is also possible to estimate sensitivity of 
the receiver for different modulation formats using the relation between SNR and BER through EVM assuming an Additive White Gaussian Noise (AWGN) channel.

For very high SNRs, the amplitude of the received signal is high enough to degrade demodulation quality due to the distortion. However, the relation between signal strength and EVM is not straightforward. Therefore, we use MATLAB simulations to model nonlinearity impairments of the MIMO receiver. To do so, our MIMO receiver is modeled using a memoryless third order polynomial nonlinearity model. using available functions in MATLAB's Communications System Toolbox, e.g., comm.MemorylessNonlinearity. EVM versus input power signal for a single carrier QAM signal with symbol rate $=0.5 \mathrm{MHz}$ is shown in Fig. 3, where our MIMO receiver has the following parameters as Gain $=32 \mathrm{~dB}$, $\mathrm{NF}_{\mathrm{SSB}}=6 \mathrm{~dB}$, and OIP3 $=22 \mathrm{dBm}$. The EVM plot includes three different regions which we will refer to as 1) noise-limited; 2) best EVM; and 3) distortion limited regions. The EVM in the flat or best EVM region is typically limited by the Phase noise of a Phase Locked Loop generating the Local Oscillator signal in practice but it is not taken into account in this model. To demodulate a signal, an EVM should be below a certain value, leading to the definition of an acceptable-EVM input power range (see Fig. 3). The EVM requirements are typically provided by standards, here we have chosen the EVM threshold of $2.5 \%(E V M=-32 \mathrm{~dB})$ for 256-QAM that meets the EVM requirements for many standards. Note that this threshold is relaxed for lower order QAMs. If input power is too low, noise will degrade EVM, if it is too high, distortion will. The width of the flat region is a measure for the dynamic range of the receiver, i.e. it improves for lower noise and higher OIP3. To allow for using power efficient low resolution ADCs, the Rx front-end must provide high enough gain to overcome the quantization noise of the ADCs, demanding a front-end with high OIP3 that we achieve in [3].

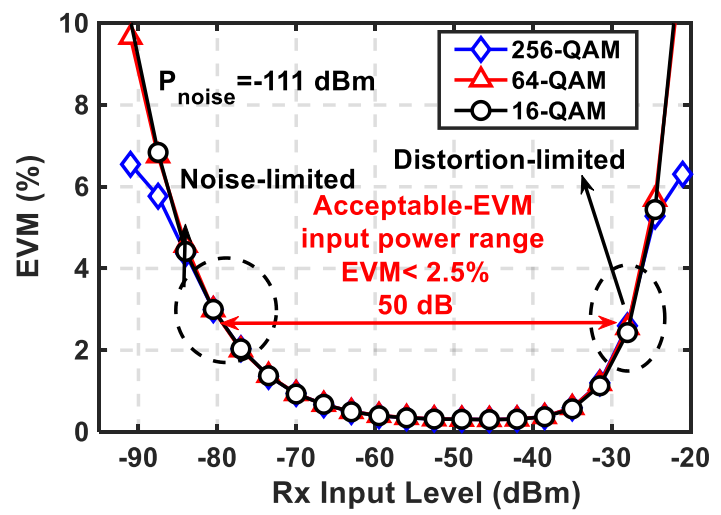

Fig. 3. MATLAB simulations modeling EVM of the Rx in [3] versus input power for 256-QAM, 64-QAM, and 16-QAM (Gain=32 dB, NF $\mathrm{SSB}=6 \mathrm{~dB}$, and OIP $3=22 \mathrm{dBm}$ ). The EVM threshold of $2.5 \%$ for 256-QAM results in $50 \mathrm{~dB}$ acceptable-EVM input power range.

\section{EXPERIMENTAL RESULTS}

\section{A. Conductive Measurements}

Measurements were performed to obtain EVM plots versus input power and evaluate the acceptable-EVM input power range. The conductive measurement set up, as shown in Fig. 4 , is used to evaluate the system performance in a controlled fashion. An spatial interference signal is produced using external vector modulators, emulating a wavefront arriving from $-90^{\circ}$ to $+90^{\circ}$. A signal-of-interest (SoI) having a broadside angle-of-arrival is emulated using power splitters. The differential receiver output is sensed using an active probe and applied to a Keysight N9030A PXA Signal Analyzer for digital demodulation by VSA software. To simplify the measurements only the I-output is used. A modulated single carrier QAM signal with $0.5 \mathrm{MHz}$ symbol rate and a root cosine pulse shaping filter with $\beta=0.35$ and Pseudo Random Binary Sequence (PRBS) data is applied. SoI Signal power is swept and EVM is measured. The resulting EVM plot is shown in Fig.5a where the sweep is repeated for 256-QAM, 64-QAM, and 16-QAM and there is no interferer. Note that the EVM performance is not affected by the order of QAM, however, the higher the QAM order, the more stringent is the EVM requirement. From the noise limited region the system noise figure is extracted to be $\mathrm{NF}_{\mathrm{SSB}}=6 \mathrm{~dB}$ where it shows a close agreement to measured noise figure of the receiver. The power level at which the distortion-limited region kicks off mainly depends on the OIP3 and the gain of the beamforming receiver. This is shown in Fig. 5b where EVM plots are depicted for three different gain values. Because OIP3 is rather independent of the receiver gain, the distortion-limited region shifts to lower input power levels as the gain increases.

The effect of co-channel interference is shown in Fig. 5c where the EVM plot is provided for both $\mathrm{CW}$ and modulated interferers having the same power $\mathrm{P}_{\mathrm{int}}=-90 \mathrm{dBm}$, which is about $20 \mathrm{~dB}$ above the noise floor. Co-channel interference degrades the EVM, reducing acceptable-EVM input power range, hence, dynamic range of the $\mathrm{Rx}$. To have EVM of less than $2.5 \%$ for 256-QAM, the SoI power has to be at least $32 \mathrm{~dB}$ above the modulated interferer level. To show the effectiveness of spatial filtering in mitigating co-channel interference, a modulated interferer having $\mathrm{P}_{\text {int }}=-70 \mathrm{dBm}$ is applied to the receiver input arriving from the same angle as SoI, e.g., broadside, so the acceptable-EVM input power range goes to zero. If the interferer arrives from an arbitrary notch angle EVM will improve. For notch angle of $+30^{\circ}$, this is shown in Fig. 5d, giving $29 \mathrm{~dB}$ improvement. This fits to the amount of spatial rejection at the $+30^{\circ}$ angle. The constellation plots for an SoI with input power of $\mathrm{P}_{\text {sig }}=-50$ $\mathrm{dBm}$ before and after spatial filtering are shown in Fig. 6a and Fig. 6b, demonstrating that digital demodulation fails in the presence of a $20 \mathrm{~dB}$ weaker interferer, while after applying spatial filtering, digital demodulation becomes possible and an EVM of $0.5 \%$ is achieved. 


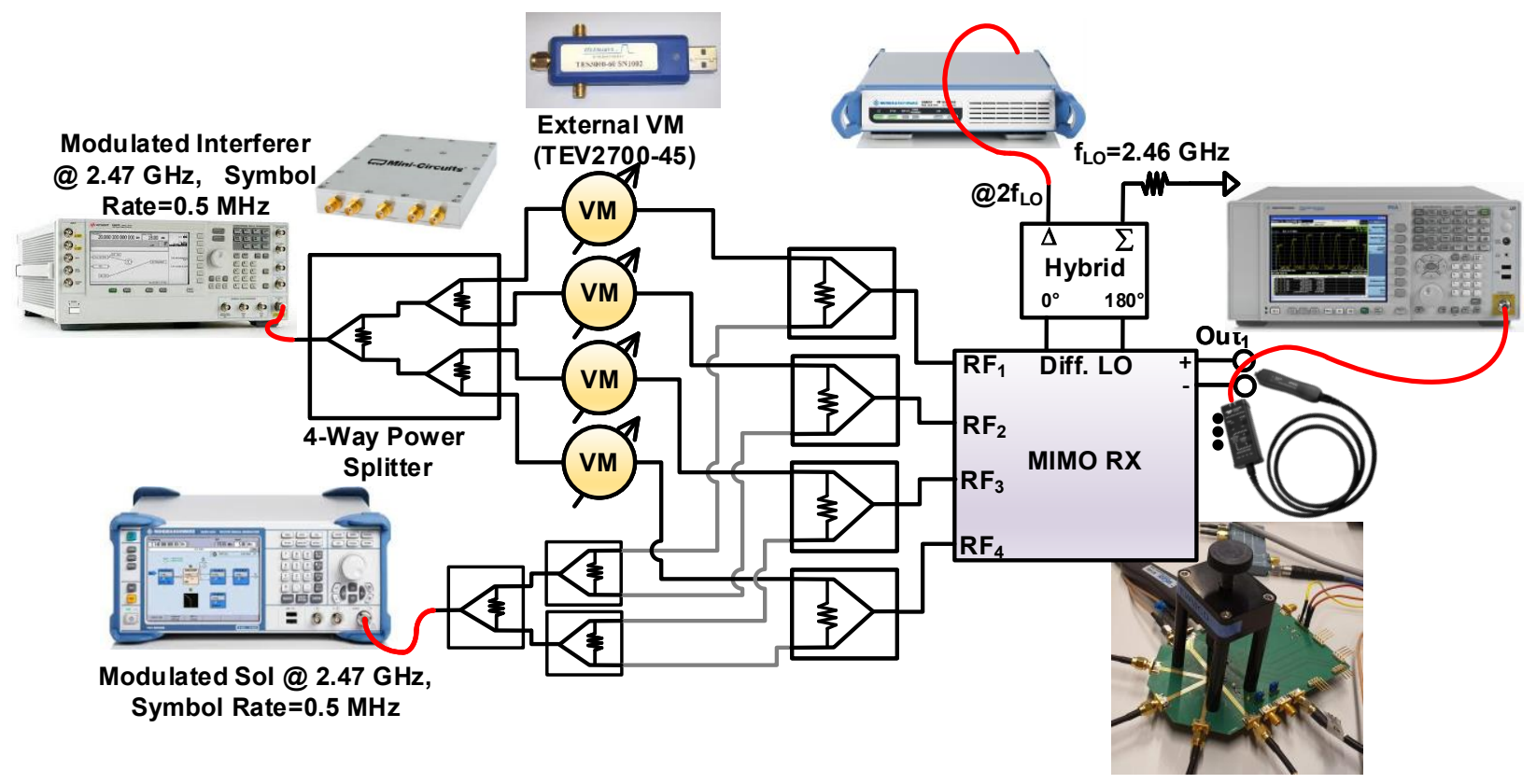

Fig. 4. Conductive measurement set-up and a 4-element antenna emulation system using external vector modulators. One interferer signal having a variable spatial angle and a SoI at fixed broadside direction are applied to the MIMO Rx chip.

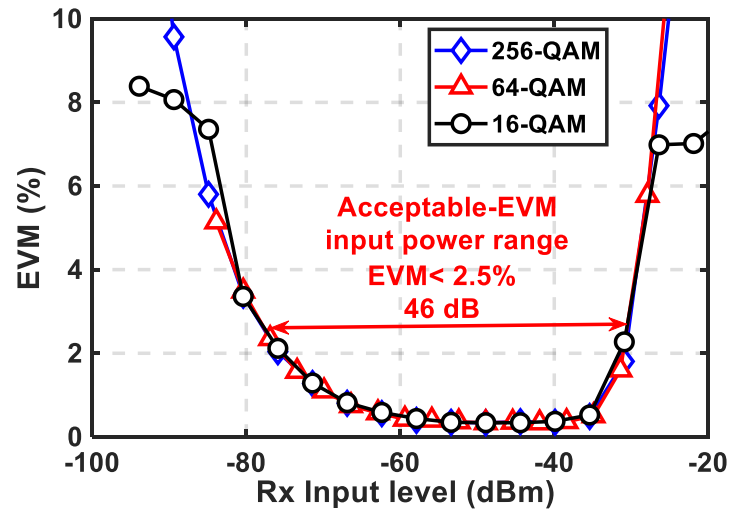

(a)

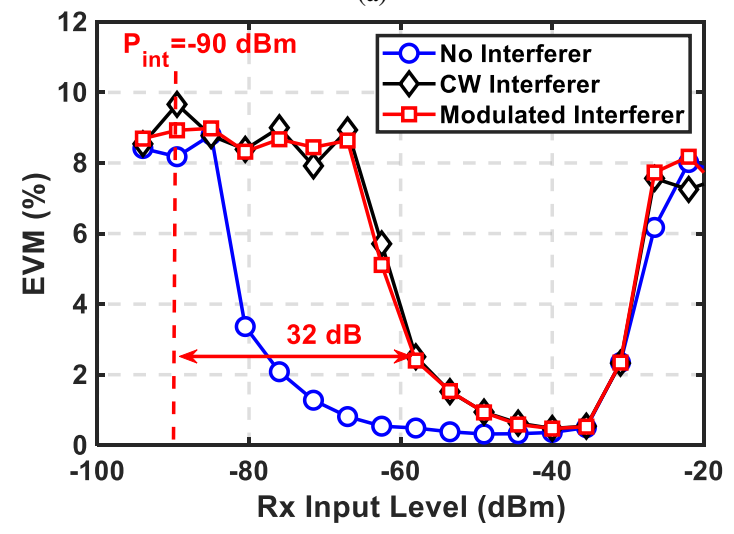

(c)

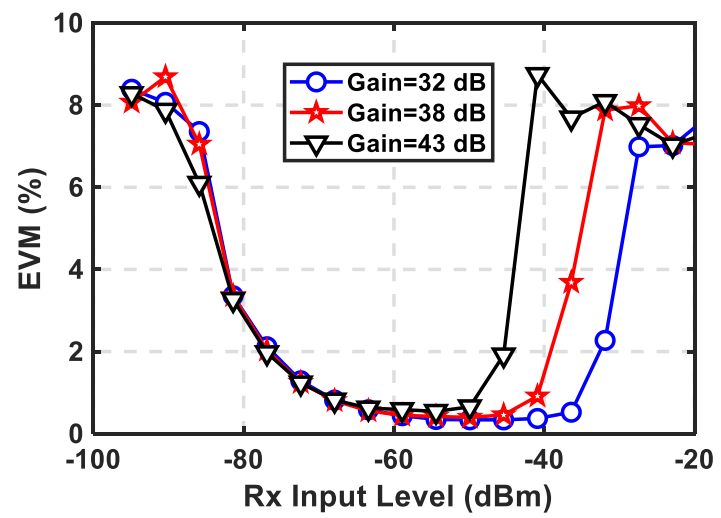

(b)

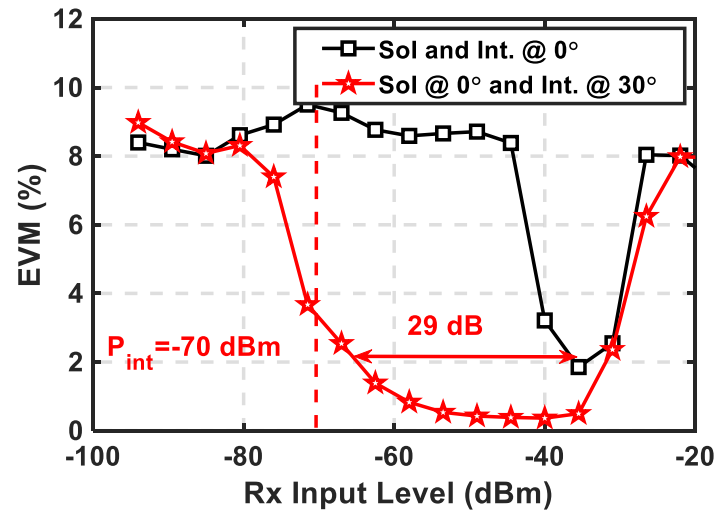

(d)

Fig. 5. (a) Measured EVM versus input power obtained from conducted measurement set up of a digitally modulated signal for 256-QAM, 64-QAM, and 16-QAM, with Gain=32 dB, NF $\mathrm{SSB}_{2}=6 \mathrm{~dB}$, OIP3=22 dBm; (b) Measured EVM for various receiver gain values (256-QAM); (c) EVM in the presence of a co-channel CW and modulated interferer (256-QAM); (d) EVM for 256-QAM in the presence of a co-channel interferer when both SoI and interferer incident the array from broadside (no spatial filtering), and when interferer is arriving from a notch at $+30^{\circ}$ (spatial filtering). 


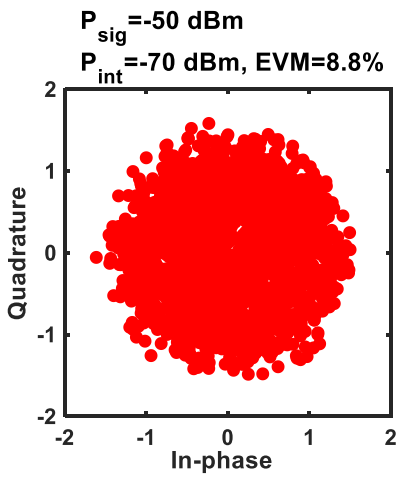

(a)

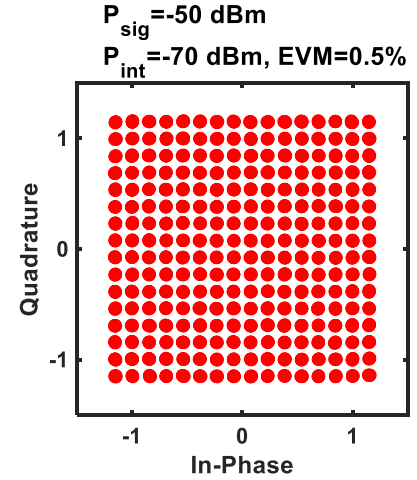

(b)
Fig. 6. (a) Constellation diagram in the presence of an interferer with $P_{\text {int }}=-70$ $\mathrm{dBm}$ when angle-of-arrival for SoI $\mathrm{P}_{\text {sig }}=-50 \mathrm{dBm}$ and interferer is the same at broadside (no spatial filtering); (b) when angle-of-arrival for the interferer is at $+30^{\circ}$ (spatial filtering).

\section{B. OTA Measurement}

OTA measurements were done with the set-up shown in Fig. 7a that uses a 4-element dipole antenna array with half-wavelength spacing in the $2.4 \mathrm{GHz}$ ISM-band, and one dipole antennas for transmitting the SoI and one antenna for the interferer. Because we are interested in effectiveness of spatial filtering for practical real world scenarios, we have performed OTA experiments in the laboratory environment but not in an anechoic chamber. First, EVM is measured when the SoI and interferer signals are combined and transmitted over-the-air with one antenna placed at broadside $0^{\circ}\left(\mathrm{P}_{\mathrm{int}}=-70\right.$ $\mathrm{dBm})$. This makes sure that both the interferer and SoI propagate to the receiver antenna array within the same propagation channel. Finally, the interferer again at $\mathrm{P}_{\text {int }}=-70$ $\mathrm{dBm}$ is transmitted using the second transmit-antenna placed at $+30^{\circ}$ for spatial filtering/separation, after which $15 \mathrm{~dB}$ extension in acceptable-EVM input power range is achieved. This result shows that less improvement is obtained compared to conductive measurements because there are variations in the received power at individual antenna array elements possibly due to multi-path propagation as well as mutual coupling between antenna elements, so the results depend on the measurement set-up. However, it is expected that better results become possible utilizing an adaptive spatial filtering solution.

\section{Conclusion}

In this paper, we proposed to characterize the interference robustness of MIMO receivers featuring hybrid analog-digital beamforming using EVM. We demonstrate experimentally how spatial interference rejection can be visualized as an improvement in acceptable-EVM input power range. Results indicate an improvement up to spatial rejection amount, e.g. $29 \mathrm{~dB}$ at $+30^{\circ}$ consistent with chip measurements. For the OTA measurement $15 \mathrm{~dB}$ improvement is observed, but results heavily depend on the experimental setup. Better results should be feasible with adaptive spatial beamforming and this is the focus of future work.

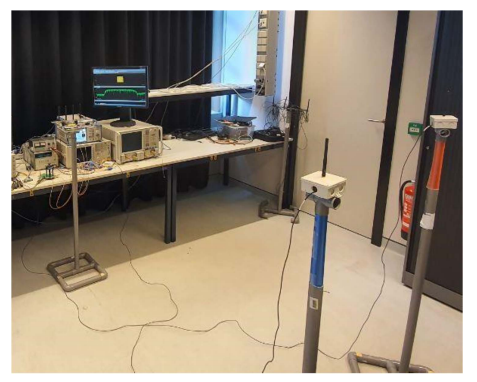

(a)

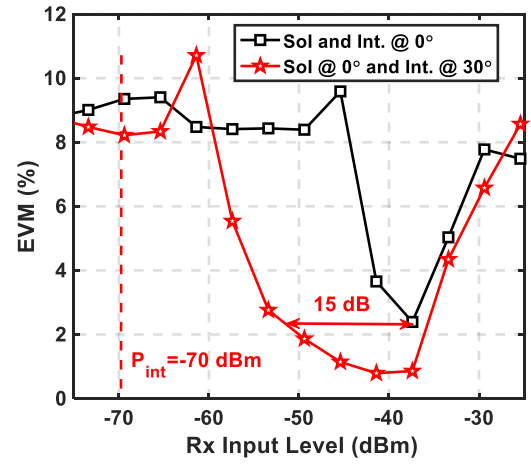

(b)

Fig. 7. (a) OTA measurement set up using a 4-element dipole antenna array with half-wavelength spacing in the $2.4 \mathrm{GHz}$ ISM-band; (b) EVM for 256-QAM in the presence of a co-channel interferer with $P_{\text {int }}=-70 \mathrm{dBm}$ when both SoI and interferer incident the array from broadside (no spatial filtering), and when interferer is arriving from a notch at $+30^{\circ}$ (spatial filtering).

\section{ACKNOWLEDGMENT}

The authors would like to thank Arnoud Rop and Gerard Wienk for measurement setup. The authors also thank Frank van Vliet, Andre Kokkeler, Pieter-Tjerk de Boer, and Masoud Abbasi Alaei for discussions. This work is part of the TTW-research program MIRABEAM with project number 14689, which is financed by the Dutch Research Council (NWO).

\section{REFERENCES}

[1] L. Zhang and H. Krishnaswamy, "A scalable 0.1-to-1.7GHz spatio-spectral-filtering 4-element MIMO receiver array with spatial notch suppression enabling digital beamforming," in IEEE Int. Solid-State Circuits Conf. (ISSCC) Dig. Tech. Papers, Jan. 2016, pp. 166-167.

[2] —, "A 0.1 -to-3.1GHz 4-element MIMO receiver array supporting analog/RF arbitrary spatial filtering," in IEEE Int. Solid-State Circuits Conf. (ISSCC) Dig. Tech. Papers, Feb. 2017, pp. 410-411.

[3] S. Golabighezelahmad, E. Klumperink, and B. Nauta, "A 1-4 GHz 4x4 MIMO Receiver with 4 Reconfigurable Orthogonal Beams for Analog Interference Rejection," in IEEE Radio Frequency Integrated Circuits Symp. (RFIC), June 2019, pp. 339-342.

[4] M. C. M. Soer, E. A. M. Klumperink, D. van den Broek, B. Nauta, and F. E. van Vliet, "Beamformer With Constant-Gm Vector Modulators and Its Spatial Intermodulation Distortion," IEEE J. Solid-State Circuits, vol. 52, no. 3, pp. 735-746, March 2017.

[5] M. D. McKinley, K. A. Remley, M. Myslinski, J. S. Kenney, D. Schreurs, and B. Nauwelaer, "EVM calculation for broadbandmodulated signals," in Proc. 64th ARFTG Conf. Dig., pp. 45-46, 2004.

[6] R. Singh, Q. Bai, T. O'Farrell, K. L. Ford, and R. J. Langley, "Demonstration of RF Digitising Concurrent Dual-Band Receiver for Carrier Aggregation over TV White Spaces," in Vehicular Technology Conference (VTC-Fall), Sept. 2016, pp. 1-5.

[7] M. E. Leinonen, M. Jokinen, N. Tervo, O. Kursu, and A. Pärssinen, "System EVM Characterization and Coverage Area Estimation of 5G Directive mmW Links," IEEE Transactions on Microwave Theory and Techniques, vol. 67, no. 12, pp. 5282-5295, Dec. 2019.

[8] M. E. Leinonen, G. Destino, O. Kursu, M. Sonkki, and A. Pärssinen, "28 GHz Wireless Backhaul Transceiver Characterization and Radio Link Budget," ETRI Journal, vol. 40, no. 1, pp. 89-100, 2018. 